\title{
New Synthetic Routes to Biologically Interesting Geranylated Acetophenones from Melicope Semecarpifolia and Their Unnatural Prenylated and Farnesylated Derivatives $^{\dagger}$
}

\author{
Likai Xia, Manchala Narasimhulu, Xin Li, Jae-Jin Shim, and Yong Rok Lee* \\ School of Chemical Engineering and Technology, Yeungnam University, Gyeongsan 712-749, Korea. *E-mail: yrlee@yu.ac.kr \\ Received November 27, 2009, Accepted December 2, 2009
}

\begin{abstract}
This paper describes a new synthetic approach for biologically interesting geranylated acetophenones. The first total syntheses of 1-(5-geranyloxy-7-hydroxy-2,2-dimethyl-2H-chromen-8-yl)ethanone and 1-[5-geranyloxy-7-hydroxy-2methyl-2-(4-methylpent-3-enyl)-2H-chromen-8-yl]ethanone, isolated from Melicope semecarpifolia, were carried out starting from commercially available 2,4,6-trihydroxyacetophenone.
\end{abstract}

Key Words: Geranylated acetophenone, Melicope semecarpifolia, Chalcone

\section{Introduction}

Prenylated and geranylated acetophenones are widely distributed in nature. ${ }^{1}$ Interestingly, it was reported that the presence of the prenyl and geranyl group leads to remarkable increases in certain bioactivities. ${ }^{2}$ These groups have shown to possess many physiological properties, including anti-convulsive, ${ }^{3}$ antifungal, ${ }^{4}$ antiproliferative, ${ }^{5}$ and antioxidant activities. ${ }^{6}$ Among these, two acetophenones, 4-(1'-geranyloxy)-2,6-dihydroxyacetophenone (1) and 4-(1'-geranyloxy)-2,6-dihydroxy-3-prenylacetophenone (2), with geranyl groups, were isolated from the fruits of Evodia merrillii, a small folk medicinal tree distributed throughout Taiwan (Fig. 1). ${ }^{1 \mathrm{a}, 7}$ Two other geranylated acetophenones bearing pyranyl rings, 1-(5-geranyloxy-7-hydroxy-2,2-dimethyl-2H-chromen-8-yl)ethanone (3) and 1-[5geranyloxy-7-hydroxy-2-methyl-2-(4-methylpent-3-enyl)-2 $H$-chromen-8-yl]ethanone (4), were isolated from Melicope semecarpifolia, a small- to medium-sized evergreen tree found at low altitude in Taiwan and the Philippines (Fig. 1). ${ }^{8}$ The roots of this plant have been used as a carminative in folk medicines. ${ }^{9}$ Isolated compounds from this plant have demonstrated antiplatelet aggregation ${ }^{10}$ and cytotoxic ${ }^{11}$ activities that have stimulated interest in the synthesis of naturally occurring acetophenones with geranyloxy groups on the benzene ring. Although synthetic schemes for 4-(1'-geranyloxy)-2,6-dihydroxyacetophenone (1) have been reported, starting from 2,4,6-trihydroxyacetophenone through 5 -steps, ${ }^{12}$ no syntheses of naturally occurring geranylated acetophenones 2-4 have been reported thus far.

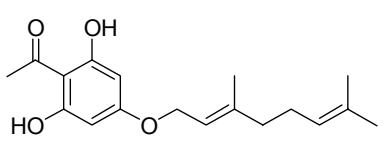

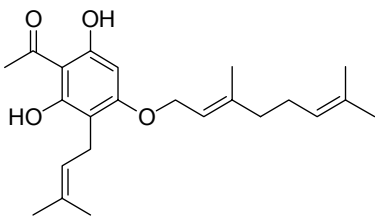

2
Recently, we reported the synthesis of biologically interesting natural products with benzopyran skeletons. ${ }^{13}$ In this lab's continuous efforts to synthesize biologically active molecules, we investigated a new route for the synthesis of biologically interesting acetophenones with geranyloxy groups on the benzene rings. Reported herein are the first total syntheses of naturally occurring geranylated acetophenones 3-4. We also report the synthesis of their unnatural prenylated and farnesylated derivatives.

\section{Results and Discussion}

Scheme 1 shows the retrosynthetic strategy for naturally occurring geranylated acetophenones $\mathbf{3}$ and $\mathbf{4}$ through regioselective $O$-geranylation reactions of key intermediates, chromenes 9 and 11. These intermediates could be prepared by EDDAcatalyzed benzopyran formation reactions of compound 7, generated from commercially available 2,4,6-trihydroxyacetophenone (5) using selective protection and deprotection reactions.

The synthesis of naturally occurring geranylated acetophenone 3 bearing the pyranyl ring was first carried out as shown in Scheme 2. Treatment of 2,4,6-trihydroxyacetophenone (5) with 2.2 eq of methoxymethyl chloride (MOMCl) and $N, N$-diisopropylethylamine in methylene chloride at room temperature for $12 \mathrm{~h}$ afforded 6 in $85 \%$ yield. ${ }^{14}$ Selective dimethoxymethylation of 5 was confirmed by ${ }^{1} \mathrm{H}-\mathrm{NMR}$ spectral analysis of the obtained compound $\mathbf{6}$. The signal for the proton of hydroxyl group of $\mathbf{6}$ was observed as a singlet associated with a hydrogen bond to a carbonyl group at $\delta 13.68 \mathrm{ppm}$. Two methoxy signals

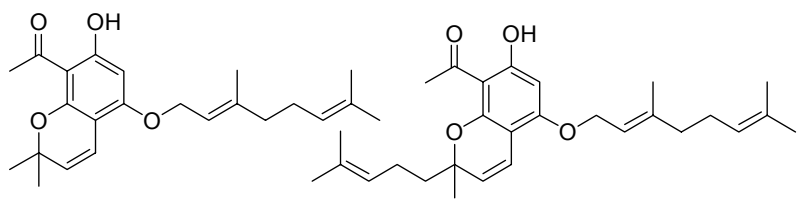

Figure 1. Naturally occurring geranylated acetophenones.

\footnotetext{
${ }^{\dagger}$ This paper is dedicated to Professor Sunggak Kim on the occasion of his honorable retirement.
} 
<smiles>CC(=O)c1c(O)cc(OC/C=C(\C)CCC=C(C)C)c2c1OC(C)(C)C=C2</smiles><smiles>CC(=O)c1c(O)cc(OCC=C(C)CCC=C(C)C)c2c1C=CC(C)(CCC=C(C)C)O2</smiles><smiles>C=C=CC=C</smiles>

Scheme 1<smiles></smiles><smiles>COc1cc(O)c(C(C)=O)c(O)c1</smiles><smiles>CC(=O)c1c(O)cc(OC/C=C(\C)CCC=C(C)C)c2c1OC(C)(C)C=C2</smiles>

3

Scheme 2<smiles>COc1cc(O)c(C(C)=O)c(O)c1</smiles>

Scheme 3

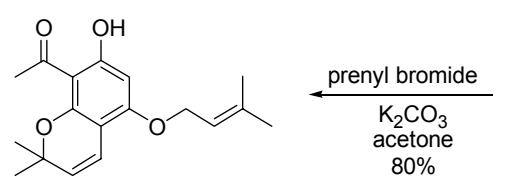

12<smiles>CC(=O)c1c(O)cc(O)c2c1OC(C)(C)C=C2</smiles>

9

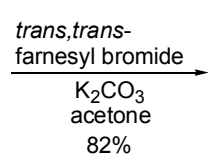

$82 \%$<smiles>CC(=O)c1c(O)cc(OCC=C(C)CCC=C(C)CCC=C(C)C)c2c1OC(C)(C)CC2</smiles>

13

Scheme 4

of the MOM ethers were observed as two singlets at $\delta 3.48$ and 3.44 ppm. Using known reaction conditions, ${ }^{14,15}$ selective orthocleavage of the methoxymethyl ether of 6 to give 7 was achieved in $80 \%$ yield by treatment with iodine in methanol at room temperature for $10 \mathrm{~h}$. Reaction of 7 with 3-methyl-2-butenal in the presence of $20 \mathrm{~mol} \%$ ethylenediamine diacetate in refluxing xylene for $10 \mathrm{~h}$ provided the desired chromene 8 in $93 \%$ yield. Deprotection of 8 with $2 \mathrm{~N} \mathrm{HCl}$ in methanol at room temperature for $10 \mathrm{~h}$ gave 9 in $51 \%$ yield. Regioselective $O$-geranylation of 9 with 1.1 eq of geranyl bromide and $\mathrm{K}_{2} \mathrm{CO}_{3}$ in acetone at room temperature for $10 \mathrm{~h}$ gave 3 in $79 \%$. The spectral data of synthetic 3 were in agreement with those reported in the literature. ${ }^{8}$

Next, the synthesis of naturally occurring geranylated aceto- phenone 4 was initiated with compound 7 as shown in Scheme 3. Reaction of 7 with citral in the presence of $20 \mathrm{~mol} \%$ ethylenediamine diacetate in refluxing xylene for $10 \mathrm{~h}$ gave cycloadduct $\mathbf{1 0}$ in $92 \%$ yield. The MOM group of $\mathbf{1 0}$ was removed by hydrolysis with $2 \mathrm{~N} \mathrm{HCl}$ in methanol at room temperature for $10 \mathrm{~h}$ to afford 11 in $47 \%$ yield. Treatment of 11 with 1.1 eq of geranyl bromide in the presence of $\mathrm{K}_{2} \mathrm{CO}_{3}$ provided the desired product 4 in $76 \%$ yield. The spectral data of compound 4 were in agreement with those reported in the literature. ${ }^{8}$

As an application of this synthetic approach, the synthesis of the unnatural acetophenones $\mathbf{1 2}$ and $\mathbf{1 3}$ with the prenyl and farnesyl groups was carried out as shown in Scheme 4. Treatment of 9 with 1.1 eq of prenyl bromide in the presence of $\mathrm{K}_{2} \mathrm{CO}_{3}$ in 

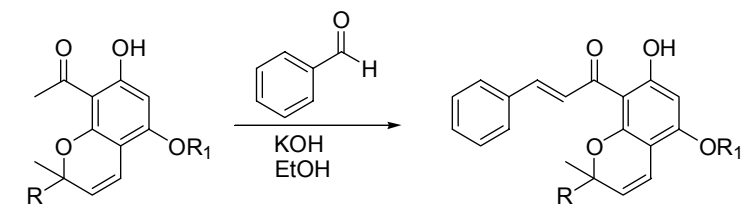

$3 \mathrm{R}=\mathrm{CH}_{3}, \mathrm{R}_{1}=$ geranyl

$4 \mathrm{R}=\mathrm{CH}_{2} \mathrm{CH}_{2} \mathrm{CHC}\left(\mathrm{CH}_{3}\right)_{2}$,

$\mathrm{R}_{1}=$ geranyl

$12 \mathrm{R}=\mathrm{CH}_{3}, \mathrm{R}_{1}=$ prenyl

$13 \mathrm{R}=\mathrm{CH}_{3}, \mathrm{R}_{1}=$ farnesyl

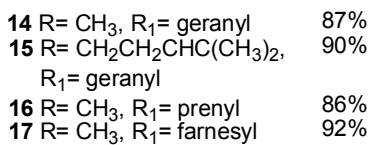

Scheme 5

acetone at room temperature for $12 \mathrm{~h}$ afforded $\mathbf{1 2}$ in $80 \%$ yield, whereas reaction with trans,trans-farnesyl bromide at room temperature for $12 \mathrm{~h}$ afforded 13 in $82 \%$ yield.

As other application, the synthesis of chalcones with prenyl, geranyl, and farnesyl group was next attempted by aldol condensation (Scheme 5). Chalcones with prenyl and geranyl groups are subclass of the flavonoids and are widely found in nature. ${ }^{16}$ They have been shown to have a range of biological activities. ${ }^{17}$ These important biological properties led to the synthesis of chalcones with prenyl, geranyl, and farnesyl group. Reactions of 3 and 4 with benzaldehyde in ethanolic $\mathrm{KOH}$ at room temperature for $48 \mathrm{~h}$ afforded chalcones 14 and 15 in 87 and 90\% yields, respectively, whereas those of $\mathbf{1 2}$ and $\mathbf{1 3}$ gave $\mathbf{1 6}$ and $\mathbf{1 7}$ in 86 and $92 \%$ yields, respectively. These reactions present a rapid route for the synthesis of pyranoacetophenones and pyranochalcones with the prenyl, geranyl, and farnesyl groups.

In conclusion, an efficient synthetic route for biologically interesting geranylated acetophenones is described. The total syntheses of geranylated acetophenones bearing the pyranyl ring, 1-(5-geranyloxy-7-hydroxy-2,2-dimethyl-2H-chromen-8-yl) ethanone and 1-[5-geranyloxy-7-hydroxy-2-methyl-2-(4-methylpent-3-enyl)-2H-chromen-8-yl]ethanone, were accomplished starting from commercially available 2,4,6-trihydroxyacetophenone through selective protection and deprotection, benzopyran formation, and $O$-geranylation. This synthetic approach was applied successfully to the syntheses of unnatural prenylated, geranylated, and farnesylated acetophenones and chalcones bearing the pyranyl ring.

\section{Experimental}

All experiments were carried out in a nitrogen atmosphere. Merck, pre-coated silica gel plates (Art. 5554) with a fluorescent indicator were used for analytical TLC. Flash column chromatography was performed using silica gel 9385 (Merck). ${ }^{1} \mathrm{H}$ and ${ }^{13} \mathrm{C}$ NMR spectra were recorded on a Bruker Model ARX (300 and $75 \mathrm{MHz}$, respectively) spectrometer in $\mathrm{CDCl}_{3}$ as the solvent chemical shift. IR spectra were recorded on a Jasco FTIR 5300 spectrophotometer.

2-Hydroxy-4,6-bis-methoxymethoxyacetophenone (6). Methoxymethyl chloride $(0.354 \mathrm{~g}, 4.40 \mathrm{mmol})$ was added to a solution of $5(0.336 \mathrm{~g}, 2.0 \mathrm{mmol})$ and diisopropylethylamine $(1.292 \mathrm{~g}, 10.0 \mathrm{mmol})$ in dry $\mathrm{CH}_{2} \mathrm{Cl}_{2}(30 \mathrm{~mL})$. The reaction mixture was stirred at room temperature for $12 \mathrm{~h}$ and then water $(30 \mathrm{~mL})$ was added. The reaction mixture was extracted with $\mathrm{CH}_{2} \mathrm{Cl}_{2}(3 \times 30 \mathrm{~mL})$. The combined organic extracts were wash- ed with saturated $\mathrm{NH}_{4} \mathrm{Cl}$ solution $(30 \mathrm{~mL})$ and evaporated in vacuo. Flash chromatography on silica gel with hexane/EtOAc (7:1) afforded $6(0.436 \mathrm{~g}, 85 \%)$ as a solid: $\mathrm{mp} 42-43{ }^{\circ} \mathrm{C} ;{ }^{1} \mathrm{H} \mathrm{NMR}$ $\left(300 \mathrm{MHz}, \mathrm{CDCl}_{3}\right) \delta 13.68(1 \mathrm{H}, \mathrm{s}), 6.22(1 \mathrm{H}, \mathrm{s}), 6.21(1 \mathrm{H}, \mathrm{s})$, $5.22(2 \mathrm{H}, \mathrm{s}), 5.14(2 \mathrm{H}, \mathrm{s}), 3.48(3 \mathrm{H}, \mathrm{s}), 3.44(3 \mathrm{H}, \mathrm{s}), 2.62(3 \mathrm{H}, \mathrm{s})$; ${ }^{13} \mathrm{C} \mathrm{NMR}\left(75 \mathrm{MHz}, \mathrm{CDCl}_{3}\right) \delta 203.3,167.0,163.6,160.5,107.1$, 97.3, 94.7, 94.2, 56.8, 56. 6, 33.1; IR (KBr) 2959, 2933, 1624, $1434,1366,1273,1224,1156,1066,1023,928,831 \mathrm{~cm}^{-1}$.

2,6-Dihydroxy-4-methoxymethoxyacetophenone (7). Iodine (152 $\mathrm{mg}, 0.60 \mathrm{mmol}$ ) was added to a solution of compound 6 (384 mg, $1.50 \mathrm{mmol})$ in methanol $(10 \mathrm{~mL})$. The reaction mixture was stirred at room temperature for $10 \mathrm{~h}$. The reaction mixture was quenched with aqueous $\mathrm{Na}_{2} \mathrm{~S}_{2} \mathrm{O}_{3}(30 \mathrm{~mL})$ and extracted with ethyl acetate $(3 \times 30 \mathrm{~mL})$. The organic layer was washed with saturated $\mathrm{NaHCO}_{3}(30 \mathrm{~mL})$ and brine $(30 \mathrm{~mL})$, dried over $\mathrm{MgSO}_{4}$, filtered, and evaporated to give the residue. Flash chromatography on silica gel with hexane/EtOAc (4:1) afforded 7 (0.255 g, 80\%) as a solid: mp $108-109{ }^{\circ} \mathrm{C} ;{ }^{1} \mathrm{H}$ NMR (300 MHz, acetone- $\left.d_{6}\right) \delta 11.71(2 \mathrm{H}, \mathrm{s}), 6.07(2 \mathrm{H}, \mathrm{s}), 5.18(2 \mathrm{H}, \mathrm{s}), 3.41(3 \mathrm{H}$, s), $2.62(3 \mathrm{H}, \mathrm{s}) ;{ }^{13} \mathrm{C} \mathrm{NMR}\left(75 \mathrm{MHz}\right.$, acetone- $\left.d_{6}\right) \delta 204.3,165.2$, 164.7, 106.6, 96.1, 94.8, 56.5, 33.0; IR (KBr) 3278, 1628, 1598, $1422,1378,1310,1278,1231,1156,1074,1022,935,818$ $\mathrm{cm}^{-1}$.

1-(7-Hydroxy-5-methoxymethoxy-2,2-dimethyl-2H-chromen-8-yl)ethanone (8). To a solution of $7(233 \mathrm{mg}, 1.10 \mathrm{mmol})$ and 3-methyl-2-butenal $(110 \mathrm{mg}, 1.32 \mathrm{mmol})$ in xylene $(20 \mathrm{~mL})$ was added ethylenediamine diacetate $(40 \mathrm{mg}, 0.22 \mathrm{mmol})$ at room temperature. The reaction mixture was refluxed for $10 \mathrm{~h}$ and cooled to room temperature. Evaporation of solvent and purification by column chromatography on silica gel using hexane/ ethyl acetate (15:1) gave $8(285 \mathrm{mg}, 93 \%)$ as an oil; ${ }^{1} \mathrm{H}$ NMR $\left(300 \mathrm{MHz}, \mathrm{CDCl}_{3}\right) \delta 13.62(1 \mathrm{H}, \mathrm{s}), 6.53(1 \mathrm{H}, \mathrm{d}, J=9.9 \mathrm{~Hz}), 6.15$ $(1 \mathrm{H}, \mathrm{s}), 5.39(1 \mathrm{H}, \mathrm{d}, J=9.9 \mathrm{~Hz}), 5.16(3 \mathrm{H}, \mathrm{s}), 3.43(3 \mathrm{H}, \mathrm{s}), 2.62$ $(3 \mathrm{H}, \mathrm{s}), 1.45(6 \mathrm{H}, \mathrm{s}) ;{ }^{13} \mathrm{C} \mathrm{NMR}\left(75 \mathrm{MHz}, \mathrm{CDCl}_{3}\right) \delta 203.3,165.8$, 158.4, 156.3, 124.8, 116.5, 106.5, 103.2, 94.8, 94.1, 77.9, 56.4, $33.2,27.8$; IR (neat) 2972, 1612, 1485, 1426, 1366, 1279, 1158, $1079,954,877,825 \mathrm{~cm}^{-1}$.

1-(5,7-Dihydroxy-2,2-dimethyl-2H-chromen-8-yl)ethanone (9). To a solution of $8(250 \mathrm{mg}, 0.90 \mathrm{mmol})$ in methanol $(10 \mathrm{~mL})$ was added $2 \mathrm{~N} \mathrm{HCl}$ (10 drops) and the reaction mixture was stirred at room temperature for $10 \mathrm{~h}$. The reaction mixture was diluted with water $(20 \mathrm{~mL})$, and extracted with EtOAc $(3 \times 30 \mathrm{~mL})$. The combined organic phases were washed with saturated NaH$\mathrm{CO}_{3}$ solution, water $(30 \mathrm{~mL})$, and dried over $\mathrm{MgSO}_{4}$. Removal of solvent at reduced pressure left an oily residue, which was then purified by column chromatography on silica gel using hexane/ethyl acetate (7:1) to give $\mathbf{9}(105 \mathrm{mg}, 51 \%)$ as an oil; ${ }^{1} \mathrm{H}$ NMR (300 MHz, aetone- $\left.d_{6}\right) \delta 13.67(1 \mathrm{H}, \mathrm{s}), 9.60(1 \mathrm{H}, \mathrm{s})$, $6.58(1 \mathrm{H}, \mathrm{d}, J=9.9 \mathrm{~Hz}), 5.95(1 \mathrm{H}, \mathrm{s}), 5.52(1 \mathrm{H}, \mathrm{d}, J=9.9 \mathrm{~Hz})$, $2.62(3 \mathrm{H}, \mathrm{s}), 1.51(6 \mathrm{H}, \mathrm{s}) ;{ }^{13} \mathrm{C}$ NMR $\left(75 \mathrm{MHz}\right.$, acetone- $\left.d_{6}\right) \delta$ 203.7, 166.9, 160.6, 158.0, 125.4, 117.4, 106.2, 103.0, 96.5, 78.9, 32.3, 28.0; IR (neat) 23330, 2976, 1606, 1503, 1425, 1367, $1273,1176,1086,961,881,819 \mathrm{~cm}^{-1}$.

1-(5-Geranyloxy-7-hydroxy-2,2-dimethyl-2H-chromen-8yl)ethanone (3). Geranyl bromide ( $96 \mathrm{mg}, 0.44 \mathrm{mmol})$ in acetone $(1 \mathrm{~mL})$ was added to a solution of compound 9 (94 $\mathrm{mg}$, $0.40 \mathrm{mmol})$ and $\mathrm{K}_{2} \mathrm{CO}_{3}(276 \mathrm{mg}, 2.0 \mathrm{mmol})$ in acetone $(10 \mathrm{~mL})$. The reaction mixture was stirred at room temperature for $10 \mathrm{~h}$. 
The solvent was evaporated under reduced pressure. The residue was treated with water, acidified with a $1 \mathrm{~N} \mathrm{HCl}$ solution, and extracted with ethyl acetate $(50 \mathrm{~mL} \times 3)$. The combined organic layers were washed with brine, dried over $\mathrm{MgSO}_{4}$, filtered, and evaporated under reduced pressure. The resulting residue was purified by flash column chromatography on silica gel with hexane/EtOAc (20:1) to give compound 3 (117 mg, 79\%) as an oil: ${ }^{1} \mathrm{H}$ NMR $\left(300 \mathrm{MHz}, \mathrm{CDCl}_{3}\right) \delta 13.82(1 \mathrm{H}, \mathrm{s}), 6.58(1 \mathrm{H}, \mathrm{d}$, $J=9.9 \mathrm{~Hz}), 6.00(1 \mathrm{H}, \mathrm{s}), 5.45(1 \mathrm{H}, \mathrm{t}, J=6.3 \mathrm{~Hz}), 5.40(1 \mathrm{H}, \mathrm{d}$, $J=9.9 \mathrm{~Hz}), 5.11(1 \mathrm{H}, \mathrm{t}, J=6.6 \mathrm{~Hz}), 4.56(2 \mathrm{H}, \mathrm{d}, J=6.3 \mathrm{~Hz}), 2.66$ $(3 \mathrm{H}, \mathrm{s}), 2.18-2.04(4 \mathrm{H}, \mathrm{m}), 1.72(3 \mathrm{H}, \mathrm{s}), 1.68(3 \mathrm{H}, \mathrm{s}), 1.61(3 \mathrm{H}$, s), $1.48(6 \mathrm{H}, \mathrm{s}) ;{ }^{13} \mathrm{C} \mathrm{NMR}\left(75 \mathrm{MHz}, \mathrm{CDCl}_{3}\right) \delta 203.2,166.3$, 160.4, 156.2 141.7, 131.9, 124.3, 123.7, 118.7, 116.8, 105.8, 103.0, 93.2, 77.9, 65.5, 39.5, 33.1, 27.8, 26.3, 25.7, 17.7, 16.7; IR (neat) 3495, 2970, 2923, 1610, 1424, 1366, 1274, 1168, $1116,961,878,817 \mathrm{~cm}^{-1}$.

1-[7-Hydroxy-5-methoxymethoxy-2-methyl-2-(4-methylpent-3-enyl)-2H-chromen-8-yl]ethanone (10). To a solution of $7(170 \mathrm{mg}, 0.80 \mathrm{mmol})$ and citral $(146 \mathrm{mg}, 0.96 \mathrm{mmol})$ in xylene $(20 \mathrm{~mL})$ was added ethylenediamine diacetate $(29 \mathrm{mg}, 0.16$ $\mathrm{mmol}$ ) at room temperature. The reaction mixture was refluxed for $10 \mathrm{~h}$ and cooled to room temperature. Evaporation of solvent and purification by column chromatography on silica gel using hexane/ethyl acetate $(15: 1)$ gave $\mathbf{1 0}(255 \mathrm{mg}, 92 \%)$ as an oil; ${ }^{1} \mathrm{H}$ NMR $\left(300 \mathrm{MHz}\right.$, acetone- $\left.d_{6}\right) \delta 13.68(1 \mathrm{H}, \mathrm{s}), 6.65(1 \mathrm{H}, \mathrm{d}$, $J=9.9 \mathrm{~Hz}), 6.17(1 \mathrm{H}, \mathrm{s}), 5.55(1 \mathrm{H}, \mathrm{d}, J=9.9 \mathrm{~Hz}), 5.29(2 \mathrm{H}, \mathrm{s})$, $5.14(1 \mathrm{H}, \mathrm{t}, J=6.3 \mathrm{~Hz}), 3.46(3 \mathrm{H}, \mathrm{s}), 2.67(3 \mathrm{H}, \mathrm{s}), 2.30-1.78$ $(4 \mathrm{H}, \mathrm{m}) 1.64(3 \mathrm{H}, \mathrm{s}), 1.57(\mathrm{~s}, 3 \mathrm{H}), 1.48(\mathrm{~s}, 3 \mathrm{H}) ;{ }^{13} \mathrm{C}$ NMR $(75$ $\mathrm{MHz}$, acetone- $\left.d_{6}\right) \delta 203.2,166.0,158.5,156.6,131.3,124.0$, 123.8, 116.8, 106.0, 102.9, 94.6, 94.3, 80.8, 55.8, 41.2, 32.6, 26.0, 24.9, 23.0, 16.8; IR (neat) 3728, 2967, 2920, 2862, 1612, $1590,1483,1427,1367,1279,1216,1160,1110,1059,955,888$, $826 \mathrm{~cm}^{-1}$.

1-[5,7-Dihydroxy-2-methyl-2-(4-methylpent-3-enyl)-2Hchromen-8-yl] ethanone (11). To a solution of 10 (208 mg, 0.60 $\mathrm{mmol})$ in methanol $(10 \mathrm{~mL})$ was added $2 \mathrm{~N} \mathrm{HCl}$ (10 drops) and the reaction mixture was stirred at room temperature for $10 \mathrm{~h}$. The reaction mixture was diluted with water $(20 \mathrm{~mL})$, and extracted with EtOAc $(3 \times 30 \mathrm{~mL})$. The combined organic phases were washed with saturated $\mathrm{NaHCO}_{3}$ solution, water $(30 \mathrm{~mL})$, and dried over $\mathrm{MgSO}_{4}$. Removal of solvent at reduced pressure left an oily residue, which was then purified by column chromatography on silica gel using hexane/ethyl acetate (7:1) to give $\mathbf{1 1}$ $(85 \mathrm{mg}, 47 \%)$ as an oil; ${ }^{1} \mathrm{H}$ NMR (300 MHz, acetone- $\left.d_{6}\right) \delta 13.80$ $(1 \mathrm{H}, \mathrm{s}), 9.71(1 \mathrm{H}, \mathrm{s}), 6.72(1 \mathrm{H}, \mathrm{d}, J=9.9 \mathrm{~Hz}), 6.04(1 \mathrm{H}, \mathrm{s}), 5.58$ $(1 \mathrm{H}, \mathrm{d}, J=9.9 \mathrm{~Hz}), 5.22(1 \mathrm{H}, \mathrm{t}, J=6.3 \mathrm{~Hz}), 2.72(3 \mathrm{H}, \mathrm{s}), 2.30-$ $1.78(4 \mathrm{H}, \mathrm{m}), 1.72(3 \mathrm{H}, \mathrm{s}), 1.65(3 \mathrm{H}, \mathrm{s}), 1.56(3 \mathrm{H}, \mathrm{s}) ;{ }^{13} \mathrm{C} \mathrm{NMR}$ $\left(75 \mathrm{MHz}\right.$, acetone- $\left.d_{6}\right) \delta 203.2,166.5,160.1,157.8,131.8,124.5$, $123.5,117.5,105.6,102.2,95.9,81.3,41.8,33.0,26.5,25.4$, 23.5, 17.3; IR (neat) 3728, 3303, 2971, 2928, 1609, 1501, 1429, $1368,1275,1174,1085,962,891,824 \mathrm{~cm}^{-1}$.

1-[5-Geranyloxy-7-hydroxy-2-methyl-2-(4-methylpent-3enyl)-2H-chromen-8-yl]ethanone (4). Geranyl bromide (48 mg, $0.22 \mathrm{mmol})$ in acetone $(1 \mathrm{~mL})$ was added to a solution of compound $11(60 \mathrm{mg}, 0.20 \mathrm{mmol})$ and $\mathrm{K}_{2} \mathrm{CO}_{3}(138 \mathrm{mg}, 1.0 \mathrm{mmol})$ in acetone $(10 \mathrm{~mL})$. The reaction mixture was stirred at room temperature for $10 \mathrm{~h}$. The solvent was evaporated under reduced pressure. The residue was treated with water, acidified with a $1 \mathrm{~N}$
$\mathrm{HCl}$ solution, and extracted with ethyl acetate $(50 \mathrm{~mL} \times 3)$. The combined organic layers were washed with brine, dried over $\mathrm{MgSO}_{4}$, filtered, and evaporated under reduced pressure. The resulting residue was purified by flash column chromatography on silica gel with hexane/EtOAc (20:1) to give compound 4 (67 mg, 76\%) as an oil: ${ }^{1} \mathrm{HNMR}\left(300 \mathrm{MHz}, \mathrm{CDCl}_{3}\right) \delta 13.83(1 \mathrm{H}$, s), $6.62(1 \mathrm{H}, d, J=9.9 \mathrm{~Hz}), 5.99(1 \mathrm{H}, \mathrm{s}), 5.43(1 \mathrm{H}, \mathrm{t}, J=6.6 \mathrm{~Hz})$, $5.35(1 \mathrm{H}, \mathrm{d}, J=9.9 \mathrm{~Hz}), 5.16-5.04(2 \mathrm{H}, \mathrm{m}), 4.57(2 \mathrm{H}, \mathrm{d}, J=6.6$ $\mathrm{Hz}), 2.66(3 \mathrm{H}, \mathrm{s}), 2.20-1.78(8 \mathrm{H}, \mathrm{m}), 1.72(3 \mathrm{H}, \mathrm{s}), 1.68(3 \mathrm{H}, \mathrm{s})$, $1.65(3 \mathrm{H}, \mathrm{s}), 1.61(3 \mathrm{H}, \mathrm{s}), 1.57(3 \mathrm{H}, \mathrm{s}), 1.43(3 \mathrm{H}, \mathrm{s}) ;{ }^{13} \mathrm{C} \mathrm{NMR}$ $\left(75 \mathrm{MHz}, \mathrm{CDCl}_{3}\right) \delta$ 203.2, 166.5, 160.6, 156.7, 141.9, 132.2, $132.1,123.9,123.9,123.1,118.9,117.5,105.9,102.8,93.2$, 80.8, 65.6, 41.7, 39.7, 33.3, 26.7, 26.5, 25.8, 23.3, 17.9, 17.8, 16.9; IR (neat) 2969, 2921, 1610, 1425, 1368, 1277, 1175, 1106, $961,875,818 \mathrm{~cm}^{-1}$

1-[7-Hydroxy-2,2-dimethyl-5-prenyloxy)-2H-chromen-8yl]ethanone (12). Prenyl bromide ( $82 \mathrm{mg}, 0.55 \mathrm{mmol})$ in acetone $(1 \mathrm{~mL})$ was added to a solution of compound 9 (117 mg, 0.50 $\mathrm{mmol})$ and $\mathrm{K}_{2} \mathrm{CO}_{3}(345 \mathrm{mg}, 2.5 \mathrm{mmol})$ in acetone $(10 \mathrm{~mL})$. The reaction mixture was stirred at room temperature for $12 \mathrm{~h}$. The solvent was evaporated under reduced pressure. The residue was treated with water, acidified with a $1 \mathrm{~N} \mathrm{HCl}$ solution, and extracted with ethyl acetate $(50 \mathrm{~mL} \times 3)$. The combined organic layers were washed with brine, dried over $\mathrm{MgSO}_{4}$, filtered, and evaporated under reduced pressure. The resulting residue was purified by flash column chromatography on silica gel with hexane/ EtOAc (15:1) to give compound $\mathbf{1 2}(121 \mathrm{mg}, 80 \%)$ as an oil: ${ }^{1} \mathrm{H} \mathrm{NMR}\left(300 \mathrm{MHz}, \mathrm{CDCl}_{3}\right) \delta 13.80(1 \mathrm{H}, \mathrm{s}), 6.56(1 \mathrm{H}, \mathrm{d}, J=9.9$ $\mathrm{Hz}), 5.98(1 \mathrm{H}, \mathrm{s}), 5.44(1 \mathrm{H}, \mathrm{t}, J=6.3 \mathrm{~Hz}), 5.38(1 \mathrm{H}, \mathrm{d}, J=9.9$ $\mathrm{Hz}), 4.50(2 \mathrm{H}, \mathrm{d}, J=6.3 \mathrm{~Hz}), 2.64(3 \mathrm{H}, \mathrm{s}), 1.77(3 \mathrm{H}, \mathrm{s}), 1.71(3 \mathrm{H}$, s), $1.46(6 \mathrm{H}, \mathrm{s}) ;{ }^{13} \mathrm{C} \mathrm{NMR}\left(75 \mathrm{MHz}, \mathrm{CDCl}_{3}\right) \delta 203.2,166.2$, $160.3,156.2$, 138.5, 124.3, 118.8, 116.8, 105.8, 102.9, 93.1, 77.8, 65.4, 33.1, 27.8, 27.7, 25.8, 18.2; IR (neat) 2928, 2923, $1605,1421,1374,1275,1176,1107,961,872,822 \mathrm{~cm}^{-1}$.

1-[7-Hydroxy-2,2-dimethyl-5-(E), (E)-famesyloxy)-2H-chromen-8-yl]ethanone (13). Trans, trans-farnesyl bromide (94 $\mathrm{mg}, 0.33 \mathrm{mmol})$ in acetone $(1 \mathrm{~mL})$ was added to a solution of compound 9 (70 mg, $0.30 \mathrm{mmol}$ ) and $\mathrm{K}_{2} \mathrm{CO}_{3}(207 \mathrm{mg}, 1.50$ $\mathrm{mmol})$ in acetone $(10 \mathrm{~mL})$. The reaction mixture was stirred at room temperature for $12 \mathrm{~h}$. The solvent was evaporated under reduced pressure. The residue was treated with water, acidified with a $1 \mathrm{~N} \mathrm{HCl}$ solution, and extracted with ethyl acetate $(50$ $\mathrm{mL} \times 3$ ). The combined organic layers were washed with brine, dried over $\mathrm{MgSO}_{4}$, filtered, and evaporated under reduced pressure. The resulting residue was purified by flash column chromatography on silica gel with hexane/EtOAc (20:1) to give compound $13(108 \mathrm{mg}, 82 \%)$ as a light yellow oil: ${ }^{1} \mathrm{H}$ NMR (300 $\left.\mathrm{MHz}, \mathrm{CDCl}_{3}\right) \delta 13.80(1 \mathrm{H}, \mathrm{s}), 6.55(1 \mathrm{H}, \mathrm{d}, J=9.9 \mathrm{~Hz}), 5.98(1 \mathrm{H}$, s), $5.44(1 \mathrm{H}, \mathrm{t}, J=6.3 \mathrm{~Hz}), 5.39(1 \mathrm{H}, \mathrm{d}, J=9.9 \mathrm{~Hz}), 5.16-5.02$ $(2 \mathrm{H}, \mathrm{m}), 4.54(2 \mathrm{H}, \mathrm{d}, J=6.3 \mathrm{~Hz}), 2.64(3 \mathrm{H}, \mathrm{s}), 2.16-1.95(8 \mathrm{H}, \mathrm{m})$, $1.71(3 \mathrm{H}, \mathrm{s}), 1.65(3 \mathrm{H}, \mathrm{s}), 1.58(6 \mathrm{H}, \mathrm{s}), 1.46(6 \mathrm{H}, \mathrm{s}) ;{ }^{13} \mathrm{C} \mathrm{NMR}$ $\left(75 \mathrm{MHz}, \mathrm{CDCl}_{3}\right) \delta 203.1,166.2,160.3,156.2,141.6,135.4$, 131.2, 124.3, 123.7, 123.5, 118.6, 116.8, 105.8, 102.9, 93.1, 77.8, 65.4, 39.6, 39.6, 39.4, 33.0, 27.8, 26.6, 26.1, 25.7, 17.6, 16.7, 16.0; IR (neat) 2969, 2920, 2857, 1700, 1610, 1428, $1366,1276,1168,1117,961,879,817,775,689,630,582$, $476 \mathrm{~cm}^{-1}$.

1-(5-Geranyloxy-7-hydroxy-2,2-dimethyl-2H-chromen-8- 
yl)-3-(E)-phenylpropenone(14). To a solution of 3 (33 mg, 0.09 $\mathrm{mmol})$ in ethanol $(10 \mathrm{~mL})$ was added $\mathrm{KOH}(50 \mathrm{mg}, 0.9 \mathrm{mmol})$ and benzaldehyde (11 mg, $0.10 \mathrm{mmol})$. The reaction mixture was stirred at room temperature for $48 \mathrm{~h}$. Evaporation of ethanol, addition of $\mathrm{NH}_{4} \mathrm{Cl}$ solution $(40 \mathrm{~mL})$, extraction with EtOAc $(3 \times$ $30 \mathrm{~mL})$, washing with brine $(30 \mathrm{~mL})$, and removal of the solvent followed by flash column chromatography on silica gel with hexane/EtOAc (20:1) gave 14 (36 mg, 87\%) as an oil: ${ }^{1} \mathrm{H}$ NMR $\left(300 \mathrm{MHz}, \mathrm{CDCl}_{3}\right) \delta 14.21(1 \mathrm{H}, \mathrm{s}), 8.12(1 \mathrm{H}, \mathrm{d}, J=15.6 \mathrm{~Hz})$, $7.75(1 \mathrm{H}, \mathrm{d}, J=15.6 \mathrm{~Hz}), 7.61-7.58(2 \mathrm{H}, \mathrm{m}), 7.42-7.37(3 \mathrm{H}, \mathrm{m})$, $6.60(1 \mathrm{H}, \mathrm{d}, J=9.9 \mathrm{~Hz}), 6.04(1 \mathrm{H}, \mathrm{s}), 5.43(1 \mathrm{H}, \mathrm{d}, J=9.9 \mathrm{~Hz})$, $5.47-5.40(1 \mathrm{H}, \mathrm{m}), 5.08(1 \mathrm{H}, \mathrm{t}, J=6.6 \mathrm{~Hz}), 4.57(2 \mathrm{H}, \mathrm{d}, \mathrm{J}=6.6$ $\mathrm{Hz}), 2.15-1.98(4 \mathrm{H}, \mathrm{m}), 1.71(3 \mathrm{H}, \mathrm{s}), 1.66(3 \mathrm{H}, \mathrm{s}), 1.59(3 \mathrm{H}, \mathrm{s})$, $1.53(6 \mathrm{H}, \mathrm{s}) ;{ }^{13} \mathrm{C} \mathrm{NMR}\left(75 \mathrm{MHz}, \mathrm{CDCl}_{3}\right) \delta 192.7,167.3,160.7$, $155.7,142.1,141.8,135.6,131.9,130.0,128.9,128.2,127.5$, 124.4, 123.6, 118.6, 117.0, 107.0, 103.3, 93.5, 77.9, 65.5, 39.5, 27.9, 27.8, 26.2, 25.7, 17.7, 16.7; IR (neat) 2969, 2923, 2357, $1729,1637,1598,1553,1450,1417,1344,1258,1218,1159$, $1116,1039,980,881,817 \mathrm{~cm}^{-1}$.

1-[5-Geranyloxy-7-hydroxy-2-methyl-2-(4-methylpent-3enyl)-2H-chromen-8-yl]-3-(E)-phenylpropenone (15). To a solution of 4 (48 mg, $0.11 \mathrm{mmol})$ in ethanol $(10 \mathrm{~mL})$ was added $\mathrm{KOH}$ (62 mg, $1.10 \mathrm{mmol})$ and benzaldehyde (13 mg, 0.12 $\mathrm{mmol})$. The reaction mixture was stirred at room temperature for $48 \mathrm{~h}$. Evaporation of ethanol, addition of $\mathrm{NH}_{4} \mathrm{Cl}$ solution $(40 \mathrm{~mL})$, extraction with EtOAc $(3 \times 30 \mathrm{~mL})$, washing with brine $(30 \mathrm{~mL})$, and removal of the solvent followed by flash column chromatography on silica gel with hexane/EtOAc (20:1) gave 15 (52 mg, 90\%) as an oil: ${ }^{1} \mathrm{H} \mathrm{NMR}\left(300 \mathrm{MHz}, \mathrm{CDCl}_{3}\right) \delta 14.23(1 \mathrm{H}$, s), $8.10(1 \mathrm{H}, \mathrm{d}, J=15.6 \mathrm{~Hz}), 7.75(1 \mathrm{H}, \mathrm{d}, J=15.6 \mathrm{~Hz}), 7.59-7.49$ $(2 \mathrm{H}, \mathrm{m}), 7.38-7.30(3 \mathrm{H}, \mathrm{m}), 6.64(1 \mathrm{H}, \mathrm{d}, J=9.9 \mathrm{~Hz}), 6.03(1 \mathrm{H}$, s), $5.45(1 \mathrm{H}, \mathrm{t}, J=6.3 \mathrm{~Hz}), 5.39(1 \mathrm{H}, \mathrm{d}, J=9.9 \mathrm{~Hz}), 5.25-5.08$ $(2 \mathrm{H}, \mathrm{m}), 4.57(2 \mathrm{H}, \mathrm{d}, J=6.3 \mathrm{~Hz}), 2.20-1.78(8 \mathrm{H}, \mathrm{m}), 1.72(3 \mathrm{H}$, s), $1.67(3 \mathrm{H}, \mathrm{s}), 1.62(3 \mathrm{H}, \mathrm{s}), 1.60(3 \mathrm{H}, \mathrm{s}), 1.47(3 \mathrm{H}, \mathrm{s}), 1.46(3 \mathrm{H}$, $\mathrm{s}),{ }^{13} \mathrm{C} \mathrm{NMR}\left(75 \mathrm{MHz}, \mathrm{CDCl}_{3}\right) \delta 192.7,167.3,160.6,156.0$, $142.1,141.7,135.6,132.1,131.9,130.0,128.9,128.3,127.5$, 123.6, 123.1, 118.6, 117.4, 106.0, 103.0, 94.0, 93.3, 80.5, 65.5, $41.4,39.5,26.6,26.2,25.7,25.6,23.0,17.7,17.5,16.7$; IR (neat) 2965, 2922, 2858, 1729, 1633, 1597, 1553, 1449, 1416, 1343, $1280,1228,1159,1104,980,893,818 \mathrm{~cm}^{-1}$.

1-[7-Hydroxy-2,2-dimethyl-5-prenyloxy)-2H-chromen-8yl]-3-(E)-phenylpropenone (16). To a solution of 12 (48 $\mathrm{mg}$, $0.16 \mathrm{mmol})$ in ethanol $(10 \mathrm{~mL})$ was added $\mathrm{KOH}(90 \mathrm{mg}, 1.60$ $\mathrm{mmol}$ ) and benzaldehyde (19 $\mathrm{mg}, 0.18 \mathrm{mmol})$. The reaction mixture was stirred at room temperature for $48 \mathrm{~h}$. Evaporation of ethanol, addition of $\mathrm{NH}_{4} \mathrm{Cl}$ solution $(40 \mathrm{~mL})$, extraction with EtOAc $(3 \times 30 \mathrm{~mL})$, washing with brine $(30 \mathrm{~mL})$, and removal of the solvent followed by flash column chromatography on silica gel with hexane/EtOAc (20:1) gave $16(54 \mathrm{mg}, 86 \%)$ as an oil: ${ }^{1} \mathrm{H}$ NMR $\left(300 \mathrm{MHz}, \mathrm{CDCl}_{3}\right) \delta 13.80(1 \mathrm{H}, \mathrm{s}), 8.12(1 \mathrm{H}, \mathrm{d}, J=$ $15.6 \mathrm{~Hz}), 7.76(1 \mathrm{H}, \mathrm{d}, J=15.6 \mathrm{~Hz}), 7.60(2 \mathrm{H}, \mathrm{d}, J=7.8 \mathrm{~Hz})$, 7.42-7.34 (3H, m), $6.59(1 \mathrm{H}, \mathrm{d}, J=9.9 \mathrm{~Hz}), 6.04(1 \mathrm{H}, \mathrm{s}), 5.45$ $(1 \mathrm{H}, \mathrm{t}, J=6.6 \mathrm{~Hz}), 5.43(1 \mathrm{H}, \mathrm{d}, J=9.9 \mathrm{~Hz}), 4.55(2 \mathrm{H}, \mathrm{d}, J=6.6$ $\mathrm{Hz}), 1.79(3 \mathrm{H}, \mathrm{s}), 1.73(3 \mathrm{H}, \mathrm{s}), 1.53(6 \mathrm{H}, \mathrm{s}) ;{ }^{13} \mathrm{C} \mathrm{NMR}(75 \mathrm{MHz}$, $\left.\mathrm{CDCl}_{3}\right) \delta 192.8,170.9,167.3,160.6,142.1,138.6,135.9,130.0$, $128.9,128.3,127.5,124.4,118.8,117.0,106.7,103.3,93.5$, 77.9, 66.3, 27.9, 25.8, 21.0, 18.3; IR (neat) 3034, 2929, 1739, $1605,1447,1355,1235,1169,1113,1031,980,882,823,747$,
$701,635,575,491 \mathrm{~cm}^{-1}$.

1-[7-Hydroxy-2,2-dimethyl-5-(E), (E)-famesyloxy)-2H-chromen-8-yl]-3-(E)-phenylpropenone (17). To a solution of 13 (70 $\mathrm{mg}, 0.16 \mathrm{mmol})$ in ethanol $(10 \mathrm{~mL})$ was added $\mathrm{KOH}(90 \mathrm{mg}$, $1.60 \mathrm{mmol}$ ) and benzaldehyde ( $19 \mathrm{mg}, 0.18 \mathrm{mmol})$. The reaction mixture was stirred at room temperature for $48 \mathrm{~h}$. Evaporation of ethanol, addition of $\mathrm{NH}_{4} \mathrm{Cl}$ solution $(40 \mathrm{~mL})$, extraction with EtOAc $(3 \times 30 \mathrm{~mL})$, washing with brine $(30 \mathrm{~mL})$, and removal of the solvent followed by flash column chromatography on silica gel with hexane/EtOAc (50:1) gave 17 (77 mg, 92\%) as a yellow oil: ${ }^{1} \mathrm{H}$ NMR $\left(300 \mathrm{MHz}, \mathrm{CDCl}_{3}\right) \delta 13.87(1 \mathrm{H}, \mathrm{s}), 8.12(1 \mathrm{H}, \mathrm{d}$, $J=15.6 \mathrm{~Hz}), 7.76(1 \mathrm{H}, \mathrm{d}, J=15.6 \mathrm{~Hz}), 7.60(2 \mathrm{H}, \mathrm{d}, J=7.8 \mathrm{~Hz})$, $7.42-7.30(3 \mathrm{H}, \mathrm{m}), 6.60(1 \mathrm{H}, \mathrm{d}, J=9.9 \mathrm{~Hz}), 6.04(1 \mathrm{H}, \mathrm{s}), 5.44$ $(1 \mathrm{H}, \mathrm{t}, J=6.6 \mathrm{~Hz}), 5.43(1 \mathrm{H}, \mathrm{d}, J=9.9 \mathrm{~Hz}), 5.18-5.02(2 \mathrm{H}, \mathrm{m})$, $4.57(2 \mathrm{H}, \mathrm{d}, J=6.6 \mathrm{~Hz}), 2.64(3 \mathrm{H}, \mathrm{s}), 2.16-1.90(8 \mathrm{H}, \mathrm{m}), 1.72$ $(3 \mathrm{H}, \mathrm{s}), 1.66(3 \mathrm{H}, \mathrm{s}), 1.59(6 \mathrm{H}, \mathrm{s}), 1.53(6 \mathrm{H}, \mathrm{s}) ;{ }^{13} \mathrm{C}$ NMR $(75$ $\left.\mathrm{MHz} \mathrm{CDCl}_{3}\right) \delta 192.7,167.3,160.6,155.7,142.1,141.8,135.6$, $135.5,131.3,130.0,128.9,128.2,127.5,124.4,127.3,123.5$, 118.6, 117.0, 106.2, 103.3, 93.5, 77.9, 65.5, 61.4, 39.7, 39.5, 27.9, 26.7, 26.1, 25.7, 17.7, 16.7, 16.0; IR (neat) 2956, 2920, $1735,1591,1444,1346,1224,1157,1114,978,880,819,769$, $696,643,576,483 \mathrm{~cm}^{-1}$.

Acknowledgments. This research was supported by the Basic Science Research Program through the National Research Foundation of Korea (NRF) funded by the Ministry of Education, Science, and Technology (2009-0064779).

\section{References}

1. (a) Adsersen, A.; Smitt, U. W.; Simonsen, H. T.; Christensen, S. B.; Jaroszewski, J. W. Biochem. Syst. Ecol. 2007, 35, 447. (b) Hu, L.-H.; Khoo, C.-W.; Vittal, J. J.; Sim, K.-Y.Phytochemistry 2000, 53,705 .

2. Nikaido, T.; Ohmoto, T.; Kinoshita, T.; Sankawa, U.; Delle Monache, F.; Botta, B.; Tomimori, T.; Miyaichi, Y.; Shirataki, Y.; Yokoe, I.; Komatsu, M. Chem. Pharm. Bull. 1989, 37, 1392.

3. Genovese, S.; Epifano, F.; Curini, M.; Dudra-jastrzebska, M. Bioorg. Med. Chem. Lett. 2009, 19, 5419.

4. (a) Phillips, W. R.; Baj, N. J.; Gunatilaka, A. A. L.; Kingston, D. G. I. J. Nat. Prod. 1996, 59, 495. (b) Fukui, H.; Egawa, H.; Koshimizu, K.; Mitsui, T. Agr. Biol. Chem. 1973, 37, 417.

5. (a) Pisco, L.; Kordian, M.; Peseke, K.; Feist, H.; Michalik, D.; Estrada, E.; Carvalho, J.; Hamilton, G.; Rando, D.; Quincoces, J. Eur. J. Med. Chem. 2006, 41, 401.

6. Mukherjee, S.; Kumar, V.; Prasad, A. K.; Raj, H. G.; Bracke, M. E.; Olsen, C. E.; Jain, S. C.; Parmar, V. S. Bioorg. Med. Chem. 2001, 9, 337

7. (a) Chou, C.-J.; Lin, L.-C. J. Nat. Prod. 1992, 55, 795. (b) Lin, L.-C. J. Nat. Prod. 1993, 56, 926.

8. Chen, J.-J.; Cho, J.-Y.; Hwang, T.-L.; Chen, I.-S. J. Nat. Prod. 2008, 71,71 .

9. Kan, W. S. Manual of Medicinal Plants in Taiwan; Natioal Research Institute of Chinese Medicine: Taipei, Taiwan, 1970; Vol. 2, pp 374

10. (a) Chen, J.-J.; Chang, Y.-L.; Teng, C.-M.; Su, C.-C.; Chen, I.-S. Planta Med. 2002, 68, 790. (b) Chen, I.-S.; Chen, H.-F.; Cheng, M.-J.; Chang, Y.-L.; Teng, C.-M.; Tsutomu, I.; Chen, J.-J.; Tsai, I.-L. J. Nat. Prod. 2001, 64, 1143.

11. (a) Chen, J.-J.; Duh, C.-Y.; Huang, H.-Y.; Chen, I.-S. Planta Med. 2003, 69, 542. (b) Chou, H.-C.; Chen, J.-J.; Duh, C.-Y.; Huang, T.-F.; Chen, I.-S. Planta Med. 2005, 71, 1078.

12. Huang, C. S.; Zhang, Z.; Li, S. H.; Li, Y. L. Chinese Chem. Lett. 
1998, 9, 799 .

13. (a) Lee, Y. R.; Choi, J. H.; Yoon, S. H. Tetrahedron Lett. 2005, 46, 7539. (b) Lee, Y. R.; Lee, W. K.; Noh, S. K.; Lyoo, W. S. Synthesis 2006, 853. (c) Lee, Y. R.; Kim D. H. Synthesis 2006, 603. (d) Wang, X.; Lee, Y. R. Tetrahedron Lett. 2007, 48, 6275. (e) Wang, X.; Lee, Y. R. Synthesis 2007, 3044. (f) Lee, Y. R.; Xia, L. Synthesis 2007, 3240. (g) Lee, Y. R.; Kim, J. H. Synlett 2007, 2232. (h) Lee, Y. R.; Kim, Y. M. Helv. Chim. Acta 2007, 90, 2401. (i) Lee, Y. R.; Li, X.; Kim, J. H. J. Org. Chem. 2008, 73, 4313. (j). Lee, Y. R.; Xia, L. Tetrahedron Lett. 2008, 49, 3283. (k) Xia, L.; Lee, Y. R. Synlett 2008, 1643. (1) Wang, X.; Lee, Y. R. Tetrahedron 2009, 65, 10125. (m) Park, B. H.; Lee, Y. R.; Lyoo, W. S. Synthesis 2009, 2146.

14. Li, Y.; Luo, Y.; Huang, W.; Wang, J.; Lu, W. Tetrahedron Lett.
2006, $47,4153$.

15. Keith, J. M. Tetrahedron Lett. 2004, 45, 2739.

16. (a) Reddy, R. V. N.; Reddy, N. P.; Khalivulla, S. I.; Reddy, M. V. B.; Gunasekar, D.; Blond, A.; Bodo, B. Phytochemistry Lett. 2008, 1, 23. (b) Lukasedar, B.; Vajrodaya, S.; Hehenberger, T.; Seger, C.; Nagl, M.; Lutz-Kutschera, G.; Robin, W.; Greger, H.; Hofer, O. Phytochemistry 2009, 70, 1030. (c) Khalivulla, S. I.; Reddy, B. A. K.; Gunasekar, D.; Blond, A.; Bodo, B.; Murthy, M. M.; Rao, T. P. J. Asian Nat. Prod. Res. 2008, 10, 953.

17. (a) Vogel, S.; Heilmann, J. J. Nat. Prod. 2008, 71, 1237. (b) Aoki, N.; Muko, M.; Ohta, E.; Ohta, S. J. Nat. Prod. 2008, 71, 1308. (c) Jayasinghe, L.; Rupasinghe, G. K.; Hara, N.; Fujimoto, Y. Phytochemistry 2006, 67, 1353. (d) Shimizu, K.; Kondo, R.; Sakai, K.; Buabarn, S.; Dilokkunanant, U. Phytochemistry 2000, 54, 737. 